\title{
Heavy Metals/Elemental Impurities Analysis
}

National Cancer Institute

\section{Source}

National Cancer Institute. Heavy Metals/Elemental Impurities Analysis. NCI Thesaurus.

Code C133964.

Analytical procedures that determine the amount of elemental impurities in drug products or drug product components. 